\title{
SARCOME D'EWING DU ROCHER
}

\author{
N. KAFFEL, N. JLASSI, S. BEN GAMRA, R. LAHIANI, H. HAJRI, M. FERJAOUI \\ SERVICE D'ORL ET DE CHIRURGIE MAXILLO-FACIALE \\ HÔPITAL CHARLES NICOLLE. TUNIS
}

\begin{abstract}
Introduction : Le sarcome d'Ewing des rochers est rare. Depuis sa première description en 1921, seulement une dizaine de cas ont été rapportés dans la littérature.

But : le but de ce travail est de rapporter les caractéristiques cliniques et thérapeutiques du sarcome d'Ewing à localisation temporale.

Méthodes : Nous rapportons le cas d'un sarcome d'Ewing à localisation temporale chez un enfant de 16 mois, diagnostiqué et traité au service ORL de I'hôpital Charles Nicolle de Tunis.

Résultats : Le diagnostique de sarcome d'Ewing du rocher a été posé à partir d'une otorragie. La confirmation histologique a été faite après biopsie et étude immuno-histochimique. Après une chimiothérapie d'induction, une exérèse chirurgicale a été réalisée, suivie d'une chimiothérapie. Aucune récidive ni métastase à distance n'a été détectée au bout de 34 mois.

Conclusion : Même si le pronostic de ces tumeurs est mauvais du fait des métastases notamment pulmonaires et osseuses, cette tumeur à localisation crânienne peut être complètement éradiquée si une résection chirurgicale complète est réalisée, suivie d'une radio-chimiothérapie.
\end{abstract}

Mots clés : Ewing ; Craniofacial; Sarcome; enfant

\section{SUMMARY}

Introduction : Primary cranial Ewing's sarcoma is rare. This tumor was first described in 1921, since then, only a few cases have been reported in the literature.

Aim: the aim of this study is to report the clinical and therapeutic aspects of primary cranial Ewing's sarcoma.

Method: We report a case of Ewing's sarcoma of the temporal bone in a 16 months child diagnosed and treated in our department of ENT of Tunis.

Result: the diagnostic of Ewing's sarcoma was made after bleeding ear. Histological confirmation was made following biopsy. After neoadjuvant chemotherapy, the tumor was surgically excised and the patient underwent chemotherapy. Neither recurrence nor distant metastasis was noted a 34 months.

Conclusion: Although the prognosis of Ewing's sarcoma in general is often poor because of early metastasis to the lungs and/or to other bones, the same tumor occurring in the cranium can often be successfully managed by intensive therapy with radical excision and radiochemotherapy.

Keywords: Ewing's; head and neck; Sarcoma; children

\section{INTRODUCTION}

Le sarcome d'Ewing est une tumeur osseuse rare correspondant à la forme indifférenciée des tumeurs neurectodermiques primitives et périphériques. Elle a été décrite pour la première fois en 1921 par James Ewing. Avant l'âge de 20 ans, c'est la seconde tumeur osseuse maligne par ordre de fréquence (30\%) derrière l'ostéosarcome $(60 \%)$. Les sièges de prédilection sont les os longs et le pelvis. Les localisations ORL sont exceptionnelles (2 à $6 \%$ des cas). Leurs faibles incidences et les difficultés d'accès à cette région anatomique posent des problèmes diagnostiques et thérapeutiques. Nous rapportons un cas de sarcome d'Ewing localisé au niveau des rochers, au travers duquel, nous rappellerons les principales caractéristiques cliniques et radiologiques de cette tumeur dans sa localisation au niveau du rocher ainsi que les différentes modalités thérapeutiques proposées.

\section{OBSERVATION :}

II s'agit d'un enfant âgé de 16 mois, sans antécédent particulier notamment pas d'antécédents otitiques ou de notion de traumatisme auriculaire. II s'est présenté à la consultation suite à l'apparition d'une otorragie spontanée gauche. L'examen otoscopique avait mis en évidence une formation tumorale charnue comblant le conduit auditif externe gauche, saignante au contact, non battante, indolore. Par ailleurs pas de paralysie faciale périphérique et pas d'adénopathies cervicales palpables. La tomodensitométrie des rochers a révélé un processus tissulaire du conduit auditif externe, étendu à l'oreille moyenne et associé à une érosion du mur de la logette et de l'os temporal ( Fig1)

L'imagérie en résonnance magnétique (IRM) confirme la lésion tissulaire du conduit auditif externe gauche étendue à l' l'oreille moyenne avec envahissement de la paro- 
tide. Elle est en hyposignal T1, se réhaussant après inject du gadolinium. (Fig 2,3)
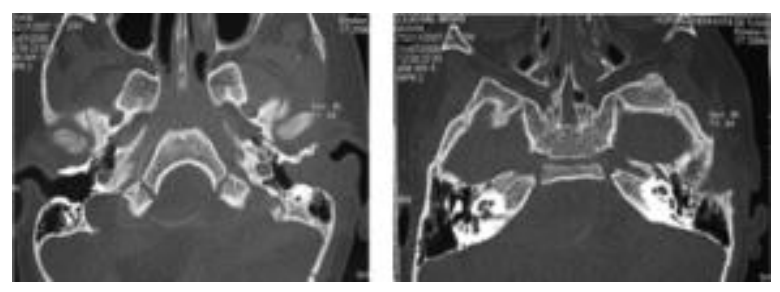

Fig 1 : TDM coupe axiale: Processus tissulaire du CAE gauche, étendu à l'OM avec érosion du mur de la logette et de l'os temporal
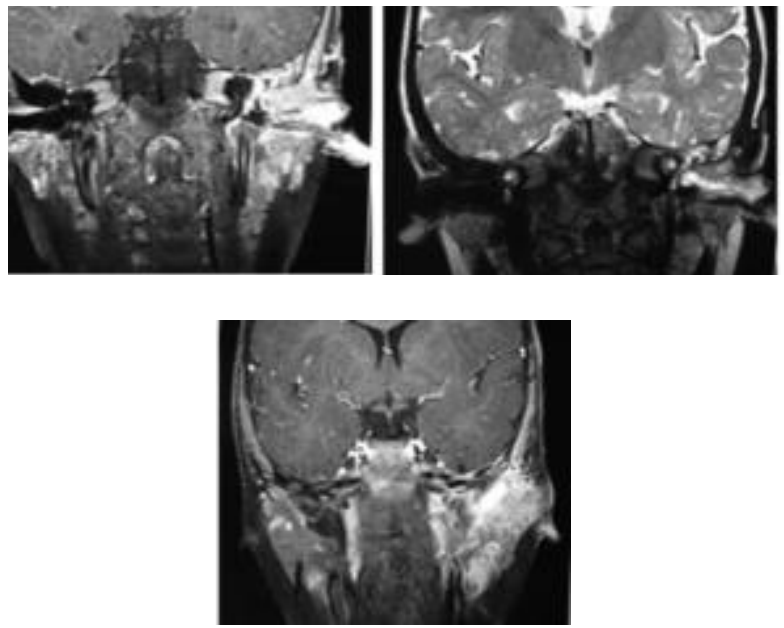

Fig 2 : IRM en coupe coronale $\mathrm{T} 1 / \mathrm{T} 2 / \mathrm{T} 1+$ gado: lésion tissulaire du CAE gauche, en hyposignal T1, se réhaussant après injection du Gadolinium

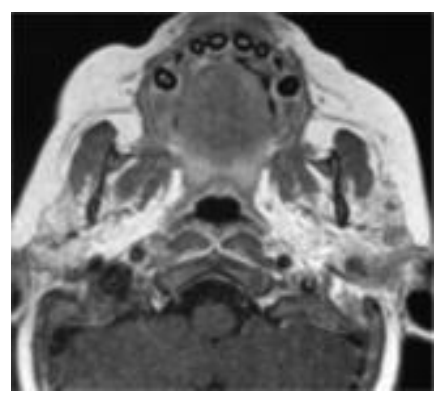

Fig. 3 : IRM en coupe axiale : masse parotidienne en hyposignal T1

Une biopsie a été faite avec étude immunohistichimique et a montré un aspect histologique de sarcome d'Ewing (petites cellules tumorales indifférenciées avec du glycogène intracytoplasmique très abondant).

Dans le cadre du bilan d'extension : une TDM thoracoabdominale, une scintigraphie osseuse et une biopsie médullaire ont été réalisées et n'ont pas révélé de localisations secondaires.

Après une approche multidisciplinaire, l'enfant a reçu tout d'abord six cures de chimiothérapie d'induction à base de Vincristine, d'Ifosfamide, de Doxorubicine et d'Etoposide. Une nouvelle IRM des rochers a montré une nette réduction du volume tumoral (plus que $80 \%$ ) autorisant alors une chirurgie d'exérèse large qui a consisté en une pétrectomie gauche, une parotidectomie exo faciale conservatrice et un curage triangulaire homolatéral. (Fig $4,5$ et 6$)$.

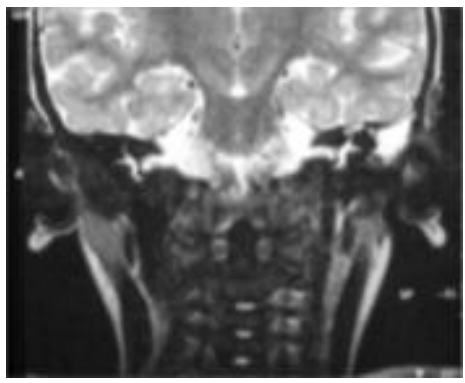

Fig 4. : IRM en coupe coronale: réduction du volume tumoral après chimiothérapie.

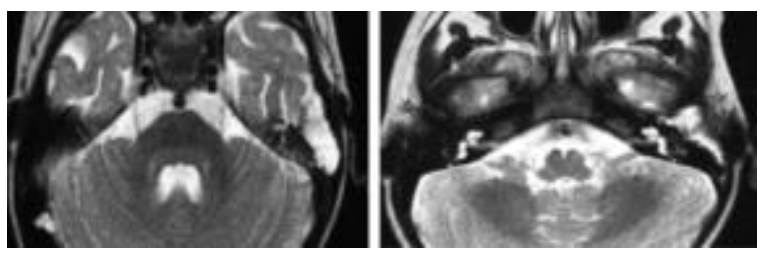

Fig. 5 : IRM en coupe axiale: diminution après chimiothérapie du comblement hyper signal du rocher, de l'oreille externe .et moyenne.

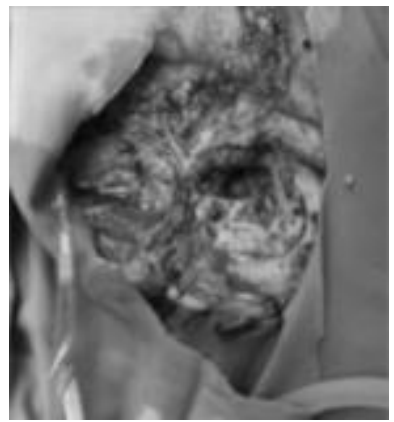

Fig. 6 : Vue per-opératoire après parotidectomie exofaciale, pétrectomie et curage ganglionnaire triangulaire

Un traitement copmlémentaire par chimiothérapie a été réalisée et le protocole a comporté 6 cures de VAI (Vincristine, Actinomycine, Ifosfamide ).

Le patient est actuellement en rémission clinique et il est asymptomatique. A un an de recul, nous n'avons aucun 
argument de récidive, ni clinique ni radiologique.

Les suites postopératoires étaient satisfaisantes notamment pas de paralysie faciale périphérique gauche.( Fig 7.)

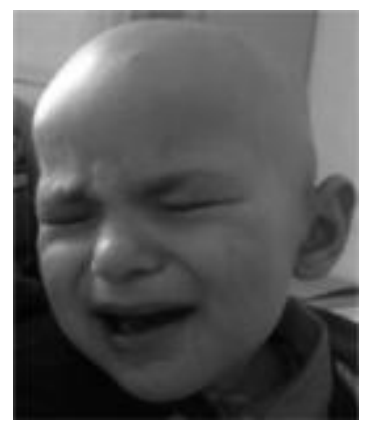

Fig. 7 : Patient à 3 mois post-opératoire avec contrôle tumoral. Pas de paralysie faciale

\section{DISCUSSION :}

C'est en 1921 que James Ewing (1866-1943) décrit cette tumeur pour la première fois, en la distinguant des lymphomes et d'autres types de cancer connus à cette époque. Le terme de "sarcome" est en fait impropre, il s'agit d'une tumeur neuroectodermique primitive (primitive neuroectodermal tumor ou PNET) (1). C'est la plus fréquente des tumeurs malignes primitives de l'os du sujet jeune après l'ostéosarcome $(10 \%$ des tumeurs osseuses). Le pic d'incidence est la deuxième décennie avec $90 \%$ des patients de moins de 20 ans et un âge médian de 14 ans. L'incidence est de 3/1.000.000 avec une prédominance masculine. Elle est six fois plus fréquente chez les enfants de race blanche que de race noire. Aucun facteur de risque n'a été identifié (2).

Le mécanisme physiopathologique le plus souvent retrouvé du sarcome d'Ewing est une translocation $t(11,22)$ responsable de l'apparition d'une protéine de fusion nommée EWS-FLI1. Cette anomalie est retrouvée dans $85 \%$ des tumeurs d'Ewing. Pour les 15\% restantes, il s'agit d'une translocation $t(21 ; 22)$ donnant lieu à la synthèse d'une protéine anormale EWS-ERG. Dans les deux cas la protéine anormale entraîne une activation continue du récepteur membranaire IGF-1, responsable de la prolifération cellulaire (3).

La tumeur peut se développer partout dans le corps, mais c'est principalement au niveau des os des membres inférieurs ( $47 \%$ : fémur, tibia, péroné) et au niveau du pelvis (29\%) qu'elles apparaissent. Certains os plats (côtes) et le rachis sont également fréquemment touchés (1). II existe une prédilection pour la localisation diaphysaire, contrairement à l'ostéosarcome qui est plus fréquemment métaphysaire.

Au niveau craniofacial, l'atteinte est rare : 3 à $6 \%$ des cas (4). C'est essentiellement au niveau du crâne et au niveau de l'appareil mandicateur où l'atteinte mandibulaire est 3 fois plus fréquente que l'atteint du maxillaire $(5$,
6). Les autres localisations craniofaciales : pétreuses, orbitaires, nasosinusiennes sont exceptionnelles et se voient dans presque $1 \%$ des cas (5).

Le sarcome d'Ewing extra osseux au niveau craniofacial peut aussi survenir mais il est encore plus exceptionnel (6). Cette forme extra squelettique ne peut pas être distinguée histologiquement du sarcome d'Ewing osseux (5). Elle se voit préférentiellement au niveau des extrémités, dans les régions paravertébrales et rétropéritonéales.

La douleur ou une tuméfaction peut révéler le diagnostic. Cependant, la symptomatologie est en fonction de la localisation de la tumeur, c'est parfois le syndrome compressif qu'elle génère qui attirera l'attention. De plus, le diagnostic peut parfois être orienté à tort vers un processus infectieux, un syndrome fébrile pouvant être présent dans $10 \%$ des cas.

Un examen clinique ORL complet, ophtalmologique, neurologique est nécessaire ainsi qu'un examen général à la recherche des métastases essentiellement pulmonaires, osseuses ou cérébrales. En effet, $30 \%$ des patients présentent déjà des métastases (au niveau des poumons ou de la moelle osseuse) au moment du diagnostic, d'où la nécessité d'un diagnostic précoce. Leur présence constitue le facteur pronostique le plus important puisque leur présence diminue considérablement la survie à cinq ans (survie à cinq ans inférieure à $20 \%$ si présence de lésions osseuses métastatiques au diagnostic contre $70 \%$ pour les formes localisées) (8).

L'imagerie est essentielle et son apport est considérable. Non seulement elle est importante pour le diagnostic et le bilan d'extension notamment au niveau de la base du crane et du parenchyme cérébral, mais elle permet aussi l'évaluation de l'efficacité du traitement et la détection de récidives. Le scanner montre une lésion tumorale ostéolytique, rehaussée par le produit du contraste. L'IRM permet essentiellement une meilleure délimitation des contours tumoraux par rapport à la réaction inflammatoire péritumorale et établiet un bilan d'extension locorégional précis (5). A l'IRM, la tumeur se présente habituellement sous la forme d'une masse en hyposignal $\mathrm{T} 1$ et un hypersignal plus ou moins hétérogène sur les séquences T2. Le bilan d'extension à distance comportera une radiographie de thorax et un scanner thoracique afin de rechercher des métastases pulmonaires (50\% des localisations métastatiques). Une scintigraphie osseuse au technétium révèlera des localisations osseuses autres que la tumeur primitive dans $25 \%$ des cas métastatiques. La recherche d'une atteinte médullaire, par myélogrammes multiples et biopsie ostéomédullaire, s'impose chez tous les patients et est positive dans $20 \%$ des cas au diagnostic (5).

Le diagnostic du SE est histologique. Il est rattaché au groupes des tumeurs neuroectodermiques primitives (PNET's) et représente avec ces groupes, des caractéristiques communes dans $88 \%$ des cas, il s'agit de similitudes 
- phénotypiques et morphologiques (tumeurs à petites cellules rondes),

- immunohistochimiques (marquage membranaire diffus par l'anticorps anti-CD99 présent dans plus de $90 \%$ des cas),

- génétiques (translocation t $(11 ; 22)(q 24 ; q 12)$.

Sur le plan microscopique, la prolifération est constituée de nappes de cellules identiques entre elles, au cytoplasme peu abondant renfermant souvent du glycogène, et à chromatine et membrane nucléaire fines. Ces plages denses et compactes de petites cellules rondes sont souvent remaniées par de la nécrose ou de l'hémorragie réalisent des images dites en pseudorosettes (5).

Le traitement du sarcome d'Ewing représente un des progrès de l'oncologie. Ce traitement est multimodal et repose sur une chimiothérapie, une résection chirurgicale et une radiothérapie permettant ainsi d'améliore le pronostic du SE et les séquelles thérapeutiques de ces localisation ORL.

Une chimiothérapie néoadjuvante est indispensable afin de traiter les micrométastases et réduire le volume de la tumeur primitive facilitant, ainsi, son exérèse chirurgicale(8). Les protocoles actuels reposent sur une polychimiothérapie associant deux à six médicaments anticancéreux parmi eux, la doxorubicine, l'actinomycine, le cyclophosphamide, l'ifosfamide, la vincristine, l'étoposide, le busulfan, le melphalan et le carboplatine. Ces associa- tions varient selon les équipes, mais les plus classiques sont : VDCA (Vincristine, doxorubicine, cyclophosphamide, actinomycine), VAIA et EVAIA (I'ifosfamide remplace la cyclophosphamide avec ou non l'adjonction d'étoposide) et enfin VIDE (Vincristine, ifosfamide, doxorubicine, étoposide) (1). Cette chimiothérapie sera suivie d'une chirurgie qui reste difficile dans les localisations ORL, souvent responsables de séquelles morphologiques et fonctionnelles importantes qui seront majorées par une radiothérapie postopératoire. La chirurgie sera suivie d'une chimiothérapie d'entretien. En cas d'extension locorégionale contre indiquant la chirurgie d'exérèse, une radiochimiothérapie sera réalisée (5).

Le pronostic a été amélioré par l'apport de la chimiothérapie pré-opératoire. La survie à 5 ans est de $60 \%$ pour les tumeurs localisées, alors qu'elle est de $20 \%$ pour les tumeurs métastatiques (6).

\section{CONCLUSION}

Le sarcome d'Ewing est tumeur osseuse agressive rapidement évolutive et métastatique. Malgré la rareté des localisations $\mathrm{ORL}$ et les difficultés de diagnostiques et thérapeutiques à ce niveau, son pronostic a été considérablement amélioré grâce à une approche multidisciplinaire.

\section{REFERENCES}

1- Taylor M., Guillon M., Champion V., Marcu M., Arnoux J.B., Hartmann O. La tumeur d'Ewing. Neurosurgy.2005; 12(9) : 1383-91 2- Parkin D.M.,. Stiller C.A, Draper G.J. et al., The international incidence of childhood cancer, Int. J. Cancer 1988; (42) : 511-20.

3-Rosai J. Ewing sarcoma. In : Ackerman's Surgical Pathology.Mosby 2000; 2:1962- 4 .

4-Watanabe H, Tsubokawa T, Katayama Y, Koyama S, Nakamura S. Primary Ewing's sarcoma of the temporal bone. Surg Neurol. 1992;37(1):54-8.

5- Velche Haag B., Dehesdin D., Proust F., Marie JP., Andrieu-Guitrancourt
J., Laquerriere A.. Sarcome d'Ewing et localisation ORL : A propos d'un cas. Ann Otolaryngol Chir Cervicofac $2002 ; 119$ : 363-8.

6 - Vaccani J. P., Forte V.,. De Jong A.L, Taylor G.. Ewing's sarcoma of the head and neck in children. International Journal of Pediatric Otorhinolaryngology. 1999; 48:209-16

7-. Erol F.S, Ozveren M.F., Ozercan I. H., Topskal C., Akdemir I. Primary Ewing's Sarcoma of the occipital bone, Neurol Med Chir (Tokyo). 2001; 41: 206-9.

8- Girma A., Paycha F., Carrier P. et al. Médecine Nucléaire. 2009;33(7) : 398-409. 